\title{
Article
}

\section{Hybrid Perovskite Terahertz Photoconductive Antenna}

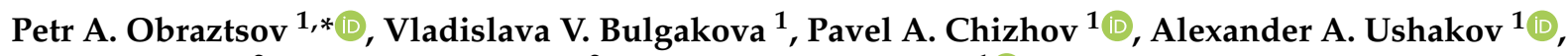 \\ Dmitry S. Gets ${ }^{2}$, Sergey V. Makarov ${ }^{2}$ and Vladimir V. Bukin ${ }^{1}$ \\ 1 Prokhorov General Physics Institute of the Russian Academy of Sciences, 119991 Moscow, Russia; \\ vbulgakova573@gmail.com (V.V.B.); pvch@inbox.ru (P.A.C.); ushakov.aleksandr@physics.msu.ru (A.A.U.); \\ vladimir.bukin@gmail.com (V.V.B.) \\ 2 Department of Physics and Engineering, ITMO University, 197101 St. Petersburg, Russia; \\ dmitry.gets@metalab.ifmo.ru (D.S.G.); s.makarov@metalab.ifmo.ru (S.V.M.) \\ * Correspondence: p.obraztsov@gmail.com
}

Citation: Obraztsov, P.A.; Bulgakova, V.V.; Chizhov, P.A.; Ushakov, A.A.; Gets, D.S.; Makarov, S.V.; Bukin, V.V. Hybrid Perovskite Terahertz Photoconductive Antenna. Nanomaterials 2021, 11, 313. https:// doi.org/10.3390/nano11020313

Academic Editor: Elias Stathatos Received: 18 December 2020

Accepted: 21 January 2021

Published: 26 January 2021

Publisher's Note: MDPI stays neutral with regard to jurisdictional claims in published maps and institutional affiliations.

Copyright: (c) 2021 by the authors. Licensee MDPI, Basel, Switzerland. This article is an open access article distributed under the terms and conditions of the Creative Commons Attribution (CC BY) license (https:/ / creativecommons.org/licenses/by/ $4.0 /)$.

\begin{abstract}
Hybrid organic-inorganic perovskites, while well examined for photovoltaic applications, remain almost completely unexplored in the terahertz $(\mathrm{THz})$ range. These low-cost hybrid materials are extremely attractive for $\mathrm{THz}$ applications because their optoelectronic properties can be chemically engineered with relative ease. Here, we experimentally demonstrate the first attempt to apply solution-processed polycrystalline films of hybrid perovskites for the development of photoconductive terahertz emitters. By using the widely studied methylammonium-based perovskites $\mathrm{MAPbI}_{3}$ and $\mathrm{MAPbBr}_{3}$, we fabricate and characterize large-aperture photoconductive antennas. The work presented here examines polycrystalline perovskite films excited both above and below the bandgap, as well as the scaling of $\mathrm{THz}$ emission with the applied bias field and the optical excitation fluence. The combination of ultrafast time-resolved spectroscopy and terahertz emission experiments allows us to determine the still-debated room temperature carrier lifetime and mobility of charge carriers in halide perovskites using an alternative noninvasive method. Our results demonstrate the applicability of hybrid perovskites for the development of scalable $\mathrm{THz}$ photoconductive devices, making these materials competitive with conventional semiconductors for $\mathrm{THz}$ emission.
\end{abstract}

Keywords: perovskite; hybrid organic-inorganic perovskite; terahertz; terahertz emission; photoconductive antenna; pump-probe spectroscopy; $\mathrm{LAPCA}^{\mathrm{MAPbI}} ; \mathrm{MAPbBr}_{3}$

\section{Introduction}

Hybrid organic-inorganic perovskites are a novel type of semiconductors demonstrating great potential for low-cost and high-performance solar cell application. The energy conversion efficiency of perovskite solar cells has skyrocketed from $3.8 \%$ to $>25 \%$ [1-4] in less than 10 years and now rivals the level of heavily developed conventional CIGS-, $\mathrm{CdTe}-$, and Si-based solar cells. Both experimental measurements and theoretical calculations show that $\mathrm{MAPbI}_{3}$ and related materials, such as $\mathrm{MAPbI}_{3}-\mathrm{xBrx}\left(\mathrm{MAPbIBr}_{3}\right)$, have bandgaps in the range of 1.1-2.1 eV, corresponding to the visible light region [5]. Their optical absorption strength is comparable to other classic semiconductors, such as GaAs, InP, and CdTe. These hybrid materials also show relatively high and balanced electron and hole mobility and very fast electron-hole pair generation. In addition, the carrier diffusion length is more than $1 \mathrm{~mm}$, implying a low concentration of deep defects [6].

The basic properties of perovskites that make them attractive for solar cells also make them appealing for terahertz $(\mathrm{THz})$ range applications $[7,8]$. Recent experiments on the generation of ultrafast photocurrents in halide perovskites excited with a femtosecond laser leading to emission of $\mathrm{THz}$ pulses have demonstrated the potential of these hybrid materials for $\mathrm{THz}$ optoelectronics [9-15]. However, while well examined in the optical range [16], hybrid perovskites remain almost completely unexplored for THz applications. Meanwhile, improvement of the efficiency of $\mathrm{THz}$ pulse sources and other $\mathrm{THz}$ optoelectronic devices 
with better designs or novel materials is one of the major goals of ongoing research in this field $[17,18]$.

Here, we experimentally demonstrate the applicability of solution-processed polycrystalline films of the hybrid perovskites $\mathrm{MAPbI}_{3}$ and $\mathrm{MAPbBr}_{3}$ for the development of large-aperture photoconductive antennas (HP-LAPCAs).

Photoconductive antennas are promising sources of $\mathrm{THz}$ radiation due to their compactness, relatively high power, and narrow directivity of the output radiation [19]. Moreover, LAPCAs are unique sources of quasi half-cycle (unipolar) THz pulses covering the lower part of the $\mathrm{THz}$ spectrum ranging from 0.1 to $1 \mathrm{THz}$ suitable for experiments on the acceleration of charged particles and electrons [20,21].

The mechanism of $\mathrm{THz}$ generation from a biased photoconductive antenna can be understood as follows: a femtosecond pulsed laser illuminates the gap of a semiconductor material and creates excited carriers in the conduction band. An external bias field accelerates the carriers up to the saturation velocity, leading to a transient photocurrent. This transient current in picosecond time domain is responsible for the $\mathrm{THz}$ pulse emission. In the far field, the $\mathrm{THz}$ electric field is proportional to the time derivative of the transient current, which can be expressed as $\mathrm{E}(t) \sim \mathrm{d} J / \mathrm{d} t$. Current density can be written as $\mathrm{J}(t)=\mathrm{N}(t) \mu \mathrm{Eb}$, where $\mathrm{N}(t)$ is the number of charge carriers, $\mu$ is the mobility of the charge carrier, and $\mathrm{Eb}$ is the applied electric field. Therefore, the efficiency and spectral characteristics of the $\mathrm{THz}$ source crucially depend on the lifetime and the achievable concentration of the excited carriers in the material.

In this report, we perform systematic investigations of ultrafast carrier dynamics in organic-inorganic halide perovskites and study the properties of $\mathrm{THz}$ radiation emitted from photoconductive antennas on their base. The work presented here examines polycrystalline perovskite films excited both above and below the bandgap, as well as the scaling of the THz emission with the applied bias field and the optical excitation fluence. The motivation for this work is to determine the suitability of hybrid perovskite LAP$\mathrm{CAs}$ for the efficient generation of $\mathrm{THz}$ pulses. We also compare the $\mathrm{THz}$ radiation from polycrystalline $\mathrm{MAPbI}_{3}$ and $\mathrm{MAPbBr}_{3}$ antennas to explicitly demonstrate that the variety of solution-processed hybrid perovskites holds a strong potential as easily scalable and chemically tunable materials for $\mathrm{THz}$ emitters. Moreover, the conducted studies allowed us to reveal the still-debated carrier lifetime and mobility of charge carriers in widely studied perovskites using an alternative noninvasive method. The developed method can be further applied to study the excited carrier transport in the variety of 3D and 2D materials having a perovskite structure.

\section{Materials and Methods}

\subsection{Perouskite Synthesis}

The synthesis from solutions containing perovskite precursors was performed using well-developed procedures [22]. Perovskite precursors and a dimethyl formamide (DMF) solvent were purchased from suppliers and used as received. The samples studied in the current work were synthesized using the following reagents: lead(II) iodide (99.99\%, trace metals basis), TCI; methylammonium iodide (99.8\%), Dyesol; lead(II) bromide (99.99\%, trace metals basis), TCI; methylammonium bromide (99.8\%), Dyesol. Glass substrates of $1.5 \times 1.5 \mathrm{~cm}^{2}$ size were subsequently ultrasonicated in acetone and 2-propanol for $5 \mathrm{~min}$, respectively, then washed with deionized water, dried with dry air, and finally cleaned with $\mathrm{O}_{3}$ for $10 \mathrm{~min}$.

Preparation of $\mathrm{MAPbBr}_{3}$ solution: $\mathrm{PbBr}_{2}(293.6 \mathrm{mg})$ and $\mathrm{MABr}(89.57 \mathrm{mg})$ were subsequently dissolved in $1 \mathrm{~mL}$ of mixture of anhydrous DMF and DMSO (7:3 ratio by volume). Preparation of $\mathrm{MAPbI}_{3}$ solution: $\mathrm{PbI}_{2}(461.01 \mathrm{mg})$ and MAI (158.97 mg) were subsequently dissolved in $1 \mathrm{~mL}$ of mixture of anhydrous DMF and DMSO (9:1 ratio by volume). All the solutions were prepared in an $\mathrm{N}_{2}$-filled glove box operating at $0.1 \mathrm{ppm}$ of both $\mathrm{O}_{2}$ and $\mathrm{H}_{2} \mathrm{O}$. 


\subsection{Thin Film Deposition}

The spin-coating cycle had the following steps: At the first step, the spin coater reached a rotational speed of $500 \mathrm{rpm}$ for $5 \mathrm{~s}$ and kept that speed for $20 \mathrm{~s}$; then it accelerated up to $3000 \mathrm{rpm}$ for $3 \mathrm{~s}$ and kept this speed for $17 \mathrm{~s}$. At the third second of the second cycle (sixth second for $\left.\mathrm{MAPbBr}_{3}\right), 0.5 \mathrm{~mL}$ of anhydrous diethyl ether was dripped onto the substrate. The films were annealed on the hot plate at $50{ }^{\circ} \mathrm{C}$ for $1 \mathrm{~min}$ and then at $100{ }^{\circ} \mathrm{C}$ for $10 \mathrm{~min}$.

The morphology of the prepared polycrystalline perovskite films was characterized using X-ray diffraction (XRD) and scanning electron microscopy (SEM) methods. Figure 1 shows the typical XRD spectra and SEM images of synthesized $\mathrm{MAPbI}_{3}$ and $\mathrm{MAPbBr}$ films.
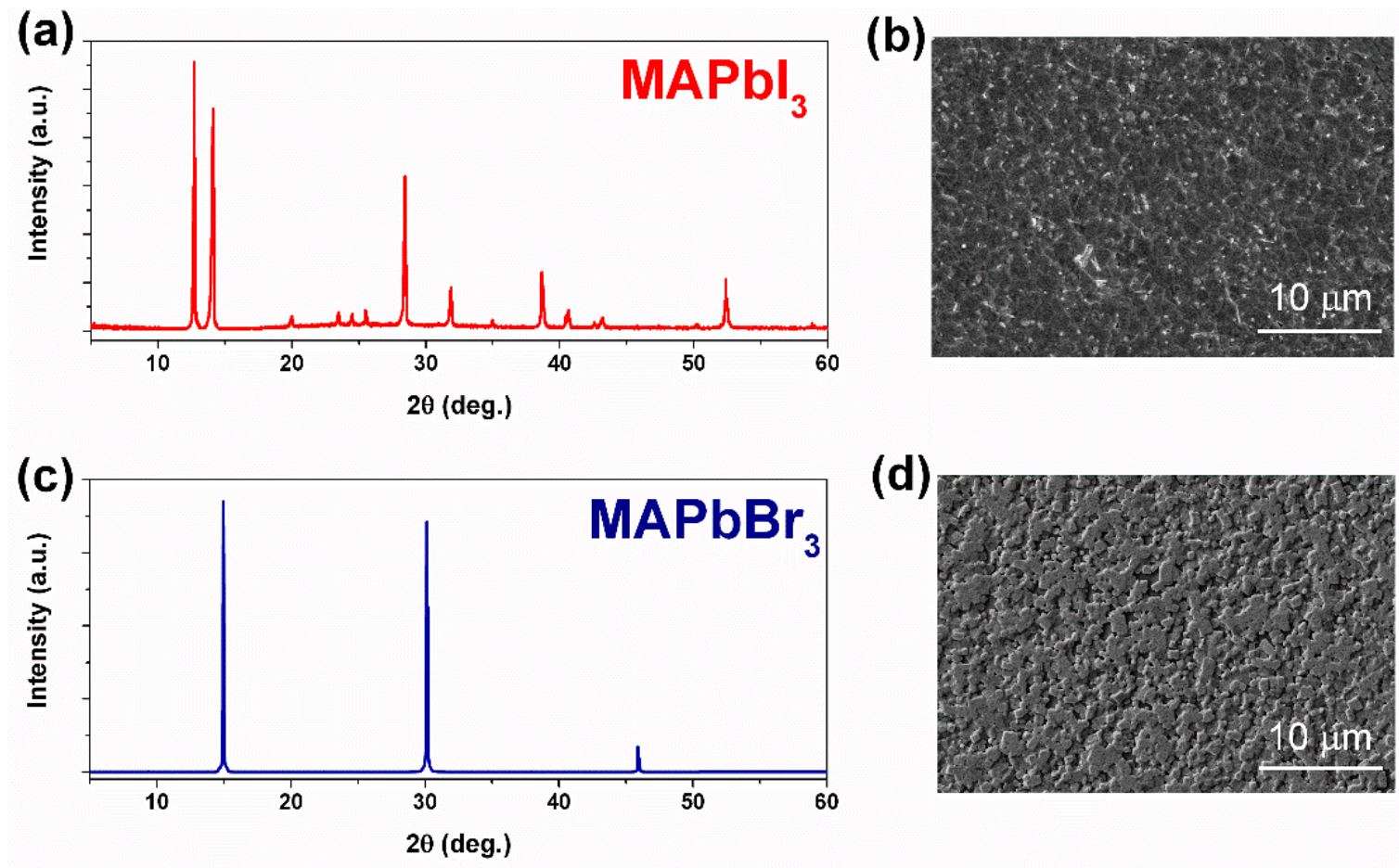

Figure 1. Typical XRD spectra and SEM images of fabricated polycrystalline $\mathrm{MAPbI}_{3}(\mathbf{a}, \mathbf{b})$ and $\mathrm{MAPbBr}_{3}(\mathbf{c}, \mathbf{d})$ films.

\subsection{Materials Characterization \\ 2.3.1. Steady-State Properties}

The linear optical absorption measurements were done using a two-channel PerkinElmer Lambda 950 spectrophotometer. To study the photoluminescent (PL) properties, we used an integrating sphere with a sample placed in the sphere center at 45 degrees relative to the incident laser beam. As a source, we used a semiconductor laser diode with a wavelength of $405 \mathrm{~nm}$ and a spot size of approximately $0.2 \mathrm{~cm}$ at a level of $1 / \mathrm{e}$.

\subsubsection{Optical Pump/THz Probe}

In optical pump/THz probe experiments, the output beam of a Ti:sapphire regenerative amplifier (Spectra-Physics Tsunami+, USA, Spitfire Pro 35-XP, $800 \mathrm{~nm}$ fundamental wavelength, $2.9 \mathrm{~mJ}$ pulse energy, $40 \mathrm{fs}$ pulse duration, $1 \mathrm{kHz}$ repetition rate, ) was split into two arms with a 70/30 beam splitter. A major portion of the laser beam was used to pump the $\mathrm{LiNbO}_{3}$ $\mathrm{THz}$ source. Here, we employed a well-known scheme for the generation of high-power coherent terahertz radiation by optical rectification of tilted-intensity-front femtosecond pulses in $\mathrm{LiNbO}_{3}$ nonlinear crystal [23]. The output laser-driven $\mathrm{THz}$ beam (used as a probe beam) was collimated and focused with polytetrafluoroethylene (PTFE) lens on the central part of the perovskite film with an intensity spot size of $\sim 1 \mathrm{~mm}$. A minor portion of the laser beam 
was directed to the sample surface without focusing to excite the perovskite film optically. The laser and $\mathrm{THz}$ beams were overlapping on the sample. The time delay between the optical and $\mathrm{THz}$ pulses at the sample position was controlled with a motorized delay line introduced in the pump optical arm. An average power of THz pulses transmitted through the optically excited perovskite sample was measured with a Golay cell detector (Tydex GC-1P, St.Petersburg, Russia). Therefore, monitoring the Golay cell output as a function of delay time between pump and probe pulses provided tracking of the temporal dynamics of pump-induced changes in the studied samples.

\subsubsection{Optical Pump/Optical Probe}

To perform the time-resolved measurements in the optical range and to reveal the relaxation rates of photoexcited carriers in perovskite $\mathrm{MAPbI}_{3}$ and $\mathrm{MAPbB}_{\mathrm{r} 3}$ films, we employed a multicolor transient absorption pump-probe setup. A detailed description of the method and setup we reported previously can be found elsewhere [24,25]. The femtosecond pulses with central wavelengths of 800 and $400 \mathrm{~nm}$ (SHG) and a femtosecond continuum were employed as pump and probe, respectively. The pump pulses were delivered by a Ti:sapphire regenerative femtosecond amplifier ( $800 \mathrm{~nm}$ wavelength, $40 \mathrm{fs}$ pulse duration, $1 \mathrm{kHz}$ repetition rate). The probe continuum pulses were generated by focusing the beam (at a wavelength of $800 \mathrm{~nm}$ and with an average power of $100 \mathrm{~mW}$ ) on a sapphire crystal. The diameter of the pump beam at the sample surface was about $500 \mu \mathrm{m}$. The femtosecond continuum was used to probe the differential transmission $(\Delta \mathrm{T} / \mathrm{T})$ in a wide spectral range spanning from 380 to $900 \mathrm{~nm}$. The pump and probe beams were polarized collinearly. A rapid motorized delay line controlled the time delay between the pump and the probe pulses. The pump-induced change of the probe transmission was detected with a spectrometer equipped with a Si CCD array (CDP ExciPro 2012). All measurements were performed at room temperature in ambient conditions.

\subsection{HP-LAPCA Fabrication}

To evaluate the performance of synthesized polycrystalline hybrid perovskites as terahertz emitters, we deposited parallel aluminum stripe-line electrodes on top of synthesized films. The gap between the electrodes for different devices varied from 2 to $4 \mathrm{~mm}$, while the width of the electrodes was $12 \mathrm{~mm}$. The perovskite thin film with conductive electrodes forms the simple-geometry large-aperture photoconductive antenna, where the perovskite material serves as a light-absorbing photoconductive substrate, while the electrodes are used to apply bias high voltage accelerating the generated photocarriers.

\section{5. $\mathrm{THz}$ Emission Experiments}

In $\mathrm{THz}$ emission experiments, the photoconductive antenna was excited by a femtosecond Ti:sapphire laser (Coherent Legend Elite, Santa Clara, CA, USA, $1 \mathrm{kHz}$ repetition rate, $130 \mathrm{fs}$ duration, $800 \mathrm{~nm}$ center wavelength, beam diameter of $10 \mathrm{~mm}$ at $\left.1 / \mathrm{e}^{2}\right)$. The antenna was excited at the fundamental $(800 \mathrm{~nm})$ and second harmonics $(400 \mathrm{~nm})$ corresponding to pump photon energies of 1.5 and $3 \mathrm{eV}$ correspondingly. To generate the second harmonic, a BBO crystal (I-type, thickness of $200 \mu \mathrm{m}$ ) was used (the energy of the second harmonic pulses was $0.3 \mathrm{~mJ}$ ). A Glan prism polarizer was used to change the energy of an optical pulse, and an optical filter was used to absorb radiation at the fundamental harmonic. Optical pumping of the perovskite part of the antenna was performed in transmission geometry from the side of the glass substrate (as shown in Figure 2). 


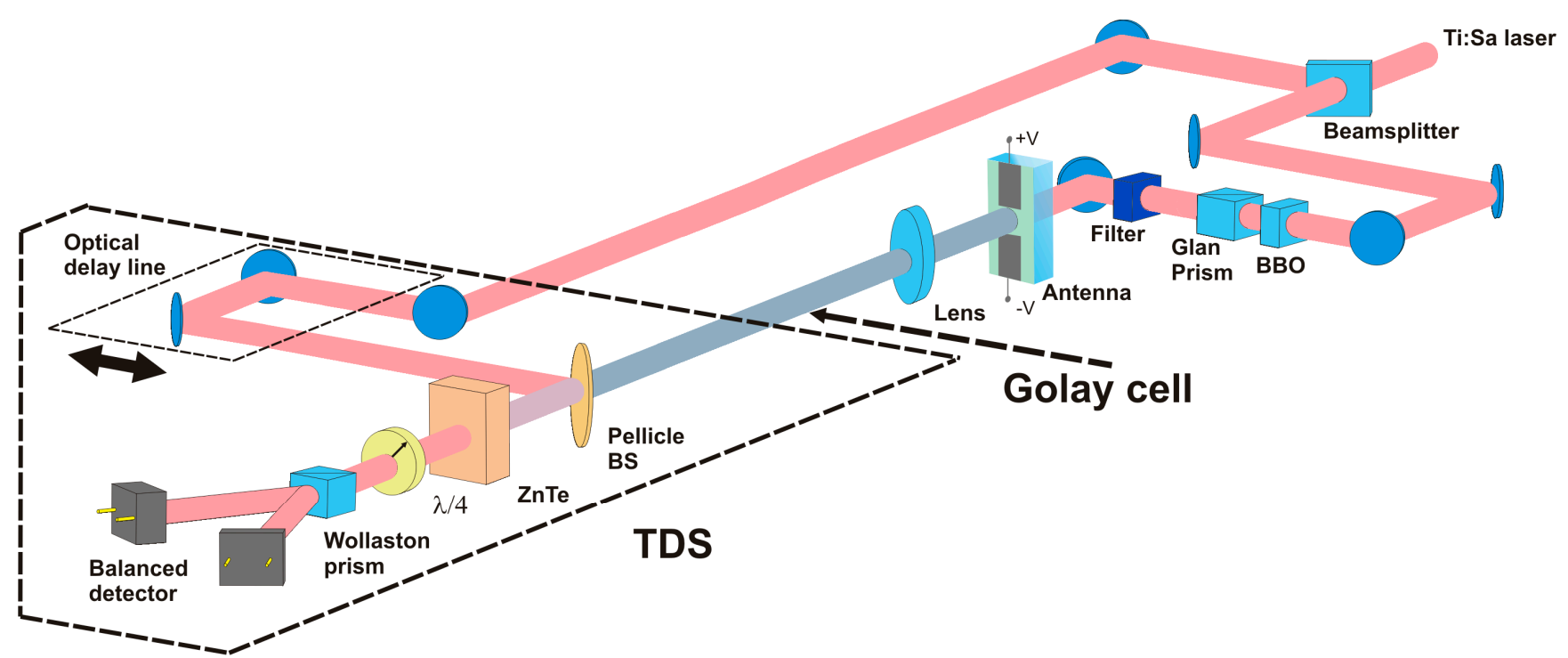

Figure 2. Experimental setup for the generation and detection of terahertz (THz) pulses from a large-aperture photoconductive antenna.

Bias high-voltage pulses with amplitudes of up to $\mathrm{Ub}=3 \mathrm{kV}$, a duration of $\sim 10 \mathrm{~ns}$, and a repetition rate of $500 \mathrm{~Hz}$ were applied to the electrodes. The repetition rate of the high-voltage pulses was $500 \mathrm{~Hz}$ to increase signal to noise ratio SNR at a frequency of $1 \mathrm{kHz}$ [26].

The terahertz waveforms were obtained using a typical time-domain spectroscopy (TDS) technique. In these experiments, the optical beam was split into two parts with a beam splitter (BS). The main part of optical radiation passes through the BS and is used to generate $\mathrm{THz}$ radiation in the antenna. Part of the radiation reflected from the BS entered the delay line for detecting the $\mathrm{THz}$ waveform. The $\mathrm{THz}$ radiation beam was focused by a PTFE lens $(\mathrm{f}=100 \mathrm{~mm})$ on an electro-optical ZnTe crystal $(0.5 \mathrm{~mm}$ thick, [110]—cut). The temporal dependence of the electric field strength of the terahertz pulse was recorded by a balanced detector with a $500 \mathrm{~Hz}$ triggered lock-in amplifier. To analyze the energy of the generated $\mathrm{THz}$ pulses, the Golay cell was used as a detector. In these experiments, laser radiation was gated at a frequency of $10 \mathrm{~Hz}$. All measurements were performed at room temperature in ambient conditions. The experimental setup is schematically shown in Figure 2.

\section{Experimental Results and Discussion}

\subsection{Steady-State Properties}

The steady-state optical properties of the fabricated polycrystalline thin films with a thickness of $\sim 600 \mathrm{~nm}$ were characterized by optical absorption and photoluminescence spectroscopies. Figure 3a shows the visible-range absorption spectra of the fabricated $\mathrm{MAPbI}_{3}$ and $\mathrm{MAPbBr}_{3}$ films. The photoluminescence spectra obtained from $\mathrm{MAPbI}_{3}$ and $\mathrm{MAPbBr}_{3}$ excited with a $400 \mathrm{~nm} \mathrm{CW}$ diode laser and the dependences of the PL peak intensity on pump fluence are shown in Figure $3 b$. 
(a)

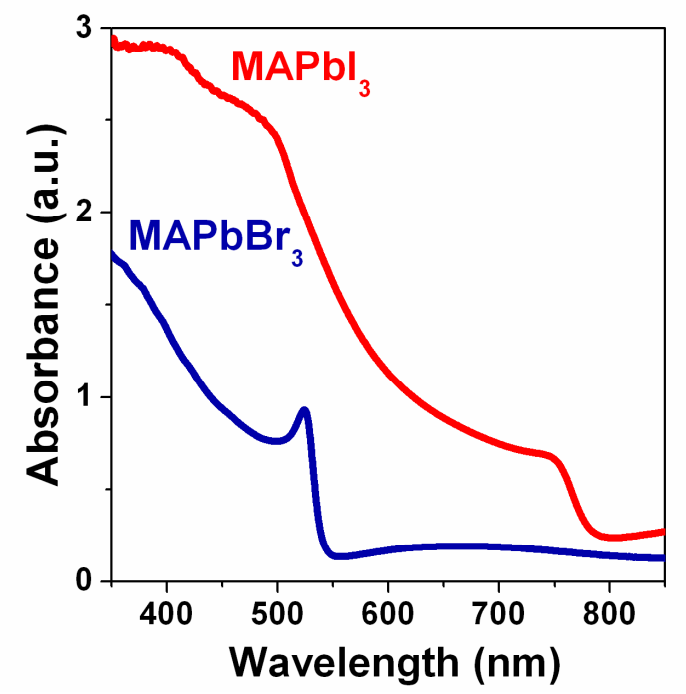

(b)

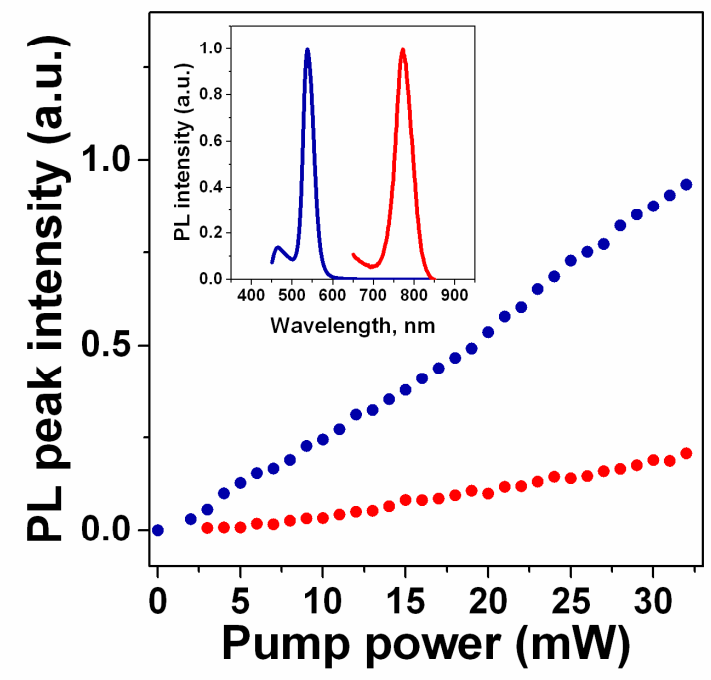

Figure 3. Steady-state optical properties of synthesized polycrystalline $\mathrm{MAPbI}_{3}$ and $\mathrm{MAPbBr}_{3}$ films placed on glass substrates. (a) Optical transmission spectra; inset shows the typical SEM image of an $\mathrm{MAPbI}_{3}$ film; (b) photoluminescence spectra obtained under excitation with a $400 \mathrm{~nm}$ laser diode; (b) the dependence photoluminescence (PL) peak intensity on pump power. Blue and red colors correspond to $\mathrm{MAPbBr}_{3}$ and $\mathrm{MAPbI}_{3}$ samples, respectively. Inset demonstrates the corresponding normalized PL spectra.

\subsection{Ultrafast Dynamics}

In order to study the dynamic response of synthesized perovskite materials and to reveal the characteristic relaxation dynamics/carrier lifetime, we performed femtosecond laser-driven optical pump/THz probe and optical pump/optical probe measurements.

The typical dynamics of an $800 \mathrm{~nm}$ pump-induced $\mathrm{THz}$ transmission change $(\Delta \mathrm{T})$ in $600 \mathrm{~nm}$ thick polycrystalline MAPbI3 is shown in Figure 4a. In Figure 4a, one can clearly see that at zero delay, the optical pumping of the sample leads to instantaneous decrease of the sample $\mathrm{THz}$ transmission related to fast photoexcited carrier generation. The $\mathrm{THz}$ absorption for a thin film is typically proportional to the photoconductivity $(\Delta \sigma)$, which in turn is proportional to the photogenerated carrier density (the number of excited carriers) $(\mathrm{N})$ and mobility $(\mu)$ following $\mathrm{E} \sim \mathrm{E} \sigma \sim \mu \mathrm{eN}$ [27].

The relaxation dynamics of the transmission change following the photoexcitation provides an estimation of the carrier lifetime in the studied material. The decay constant $\tau=360$ ps derived from the exponential fitting of the experimental data is in agreement with the previously reported data on the carrier lifetime in halide perovskites [28]. Figure $4 \mathrm{~b}$ shows the pump-induced transmission change $(\Delta T)$ as a function of pump fluence.

To reveal the relaxation rates of photoexcited carriers in perovskite films, we employed a multicolor transient absorption pump-probe setup based on the same Ti:sapphire femtosecond laser as used in optical pump/THz probe experiments. A detailed description of the method and setup can be found in the methods section. The normalized differential transmission of a $500 \mathrm{~nm}$ probe as a function of delay time between pump and probe pulses measured for both samples is presented in Figure $4 \mathrm{~b}$. Under both above and below the bandgap, optical pumping of the decreased transmission (pump-induced absorption) of the probe pulse at a wavelength of $500 \mathrm{~nm}$ can be observed. The observed transmission change is associated with the reabsorption of pump photons by the pump-induced excited carriers $[29,30]$. Therefore, the temporal dynamics and pump fluence dependence of the induced absorption provide direct information on the lifetime and created a concentration of excited carriers. Figure $4 \mathrm{~b}$ shows the pump fluence dependence of $\Delta \mathrm{T}$ measured at zero delay time for above and below the bandgap excitation of $\mathrm{MAPbI}_{3}$ and $\mathrm{MAPbBr}_{3} \mathrm{samples}_{\text {. }}$ Reasonably at lower pump fluences, corresponding to lower carrier concentrations, $\Delta \mathrm{T}$ 
linearly depends on pump fluence, which is in accordance with the optical pump/THz probe data (see Figure $4 a$ ). However, at a higher pump fluence, $\Delta \mathrm{T}$ clearly demonstrates the saturation behavior. Moreover, for both samples, absorption of pump photons having energy exceeding the bandgap of the material leads to the creation of a larger number of carriers comparing with below the bandgap excitation. Note that under $800 \mathrm{~nm}$ excitation, we do not observe a significant contribution of two-photon processes.
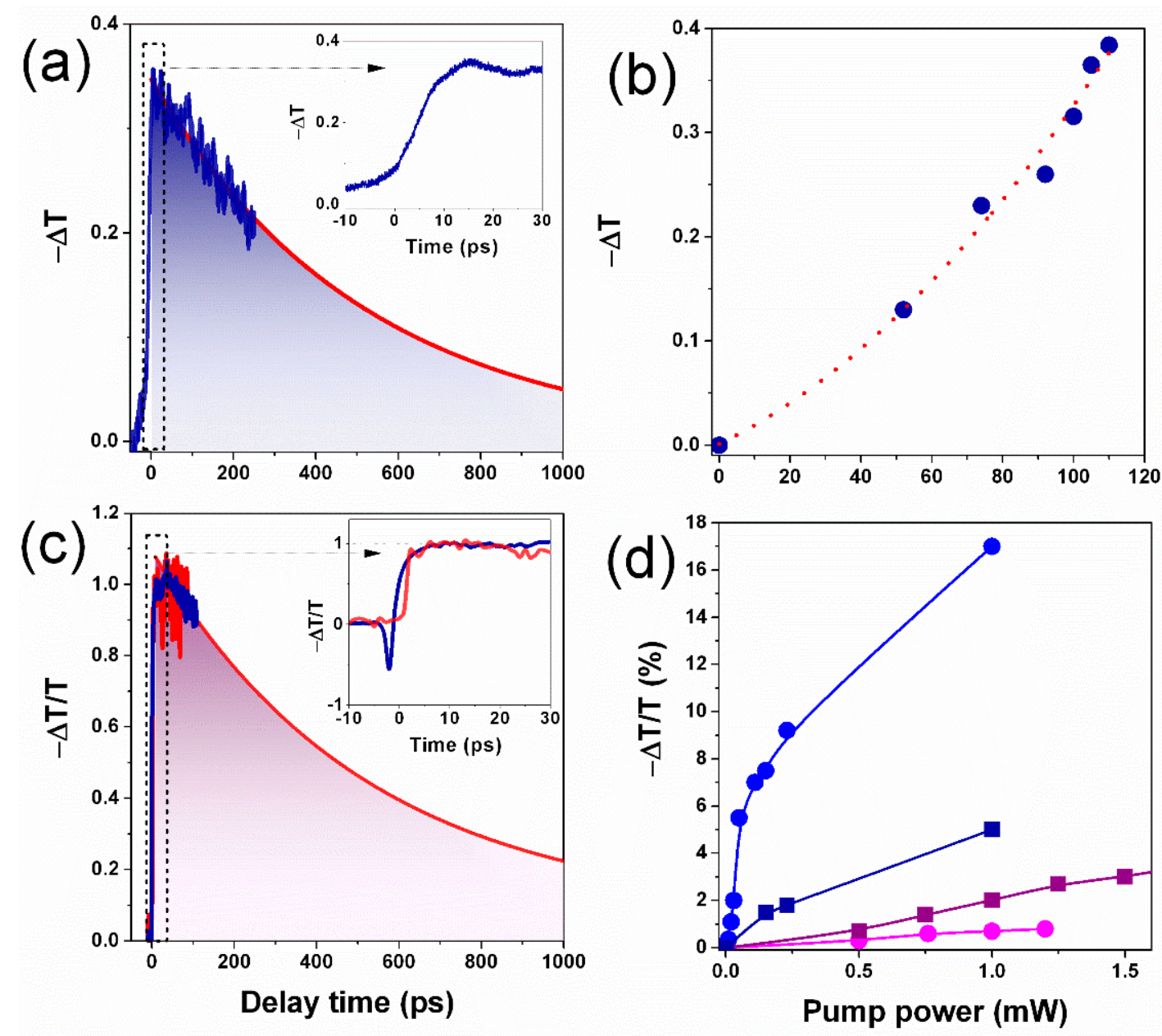

Figure 4. Temporal dynamics of the pump-induced $\mathrm{THz} /$ visible range transmission change in $\mathrm{MAPbI}_{3}(\mathrm{blue})$ and $\mathrm{MAPbBr}_{3}$ (red) films. (a) $\mathrm{THz}$ transmission change $(\Delta \mathrm{T})$ as a function of delay time between $800 \mathrm{~nm}$ pump and THz probe pulses. (c) Optical transmission change $(\Delta \mathrm{T} / \mathrm{T})$ as a function of delay time between $400 \mathrm{~nm}$ pump and $500 \mathrm{~nm}$ probe $40 \mathrm{fs}$ pulses. Exponential fitting with a decay constant $\tau=360$ ps is shown with solid red lines. (b,d) The transmission change amplitude as a function of the pump-pulse power. The data obtained under 400 and $800 \mathrm{~nm}$ excitations are shown with blue and magenta colors correspondingly.

\section{3. $\mathrm{THz}$ Emission from HP-LAPCA}

In the $\mathrm{THz}$ emission experiments, optical pumping of the perovskite part of the antenna was performed in transmission geometry from the side of the glass substrate in a way schematically shown in Figure 5a. Figure 5b-e shows the typical waveforms and corresponding frequency (FFT) spectra obtained from the fabricated LAPCAs based on $\mathrm{MAPbI}_{3}$ and $\mathrm{MAPbBr}_{3}$ perovskites under 400 and $800 \mathrm{~nm}$ optical excitations. 

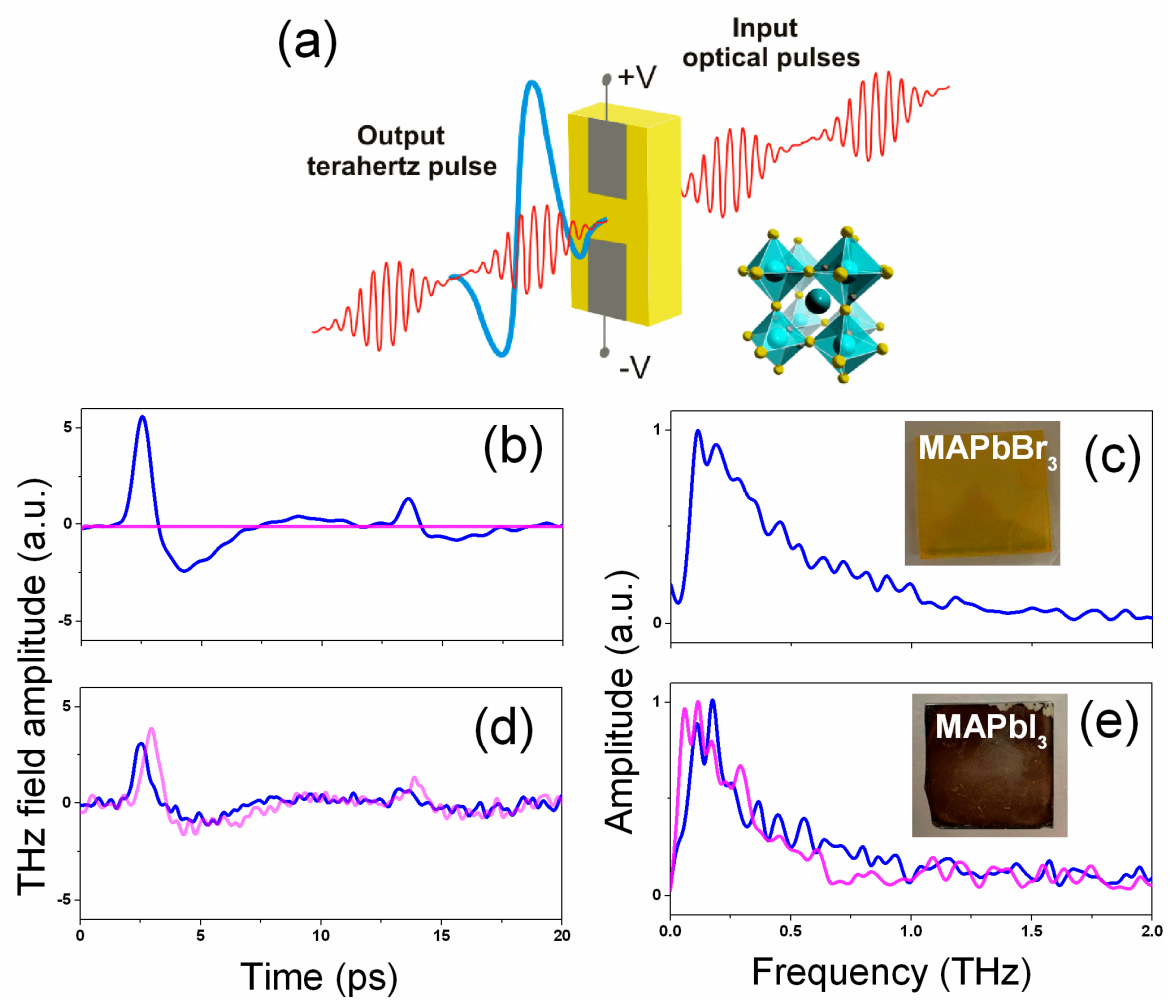

Figure 5. (a) Scheme of the THz emission from an HP-LAPCA (large-aperture photoconductive antenna). Time domain waveforms of the $\mathrm{THz}$ emission from $\mathrm{MAPbI}_{3}(\mathbf{b})$ and $\mathrm{MAPbBr}_{3}(\mathbf{d})$ and corresponding frequency domain spectra (c,e) obtained under above and below the bandgap excitation. The data obtained under 400 and $800 \mathrm{~nm}$ excitations are shown in blue and magenta colors.

Under $400 \mathrm{~nm}$ optical excitation from both materials, quasi-unipolar $\mathrm{THz}$ pulses with an full width at half maximum (FWHM) duration of $\sim 1$ ps were obtained. Under $800 \mathrm{~nm}$ excitation, $\mathrm{THz}$ pulses with similar waveforms and spectra were also obtained from an $\mathrm{MAPbI}_{3}$-based LAPCA. The obtained waveforms are typical for photoconductive antennas with a large interelectrode distance [19]. It is important to note that in such geometry of emitter, the temporal profile of the $\mathrm{THz}$ pulse is determined by the interelectrode distance rather than by the lifetime of the carriers in the photoconductive substrate.

As described in detail in the methods section, the energy of the generated $\mathrm{THz}$ pulses was measured with a Golay cell detector. The assumption of non-uniform illumination with a Gaussian intensity profile optical beam was used to analyze the data. The $\mathrm{THz}$ pulse energy was calculated using the following equation:

$$
\mathrm{W}_{\mathrm{THz}}=\beta \int_{\mathrm{S}} \mathrm{F}_{\mathrm{THz}}(\mathrm{r}) \mathrm{dS}
$$

where $\mathrm{F}_{\mathrm{THz}}$ is terahertz fluence: $\mathrm{F}_{\mathrm{THz}}(r)=\mathrm{F}(\mathrm{r}) \cdot \mathrm{\eta}(\mathrm{F}(\mathrm{r}))$, and $\mathrm{F}(\mathrm{r})=\left(2 \mathrm{~W}_{\mathrm{opt}} / \pi \mathrm{r}_{0}{ }^{2}\right) \cdot \exp \left(-2 \mathrm{r}^{2} / \mathrm{r}_{0}{ }^{2}\right)$ is optical fluence [31]. The optical-to-THz conversion efficiency is $\eta=\left(\tau \mathrm{E}_{\mathrm{b}}{ }^{2} / 2 \mathrm{~F}(\mathrm{r}) \mathrm{Z}_{0}\right) \cdot\left(\mathrm{F}(\mathrm{r}) / \mathrm{F}(\mathrm{r})+\mathrm{F}_{\text {sat }}\right)^{2}$, where $\tau$ is the THz pulse duration, $\mathrm{E}_{\mathrm{b}}$ is the electric bias field, $Z_{0}$ is the free space impedance, $F_{\text {sat }}=h v \cdot(1+n) / e \mu Z_{0}(1-R)$ is the necessary fluence for extracting half of the maximum radiated field (saturation fluence), $\mathrm{h} v$ is the photon energy of the excitation wavelength, e is the electron charge, $\mathrm{n}$ is the THz refractive index, $\mu$ is the carrier mobility, and $\mathrm{R}$ is the optical reflection of the semiconductor substrate. The THz pulse duration in calculations is $\tau=1 \mathrm{ps}$, and the size of the $10 \mathrm{~mm}$ optical beam diameter at a $1 / \mathrm{e}^{2}$ level. Integration is carried out over the area between the electrodes. The dimensionless factor $\beta$ is included in Equation (1) to take into account the attenuation of the THz pulse energy due to absorption in the antenna substrate, reflections, and so 
forth. We assume that the propagation medium of the THz-generated pulse is air due to the fact that the perovskite layer $(600 \mathrm{~nm})$ is small on the scale of the terahertz wavelength.

Figure 6 shows a comparison of the data obtained for $\mathrm{MAPbI}_{3}$ and $\mathrm{MAPbBr}_{3}$ under $400 \mathrm{~nm}$ excitation. We found that in both cases, the LAPCA's emitted terahertz energy is quadratically dependent on the applied bias field (Eb). Lower relative permittivity in combination with higher exciton energy in $\mathrm{MAPbBr}_{3}$ in comparison with $\mathrm{MAPbI}_{3}$ in turn may lead to higher efficiency of the optical-to- $\mathrm{THz}$ conversion efficiency.

(a)

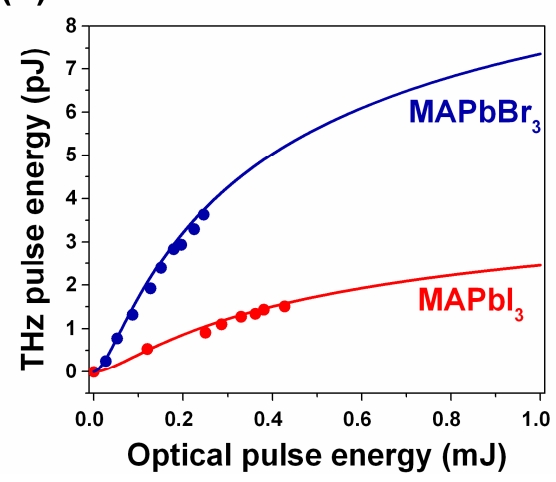

(b)

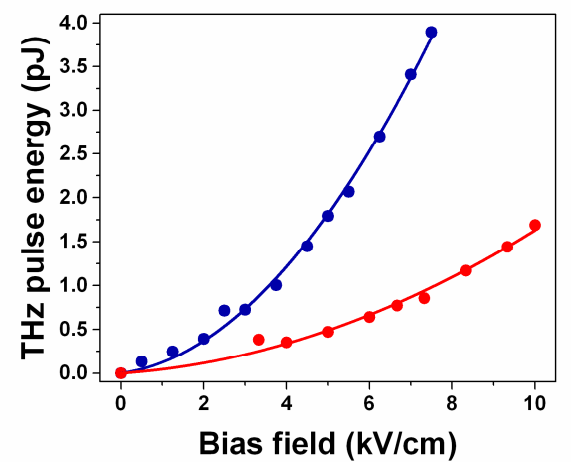

Figure 6. The terahertz pulse energy as a function of the optical excitation energy (a) and applied bias voltage (b) obtained from the HP-LAPCA optically excited above the bandgap $(400 \mathrm{~nm})$. The data obtained for HP-LAPCAs based on MAPbI 3 and $\mathrm{MAPbBr}_{3}$ are shown with red and blue colors correspondingly.

The experimental data are in good agreement with the theory [32]. Using the above data and Equation (1), we estimate the saturation fluence for both perovskite materials: $\mathrm{F}_{\text {sat }}=0.194 \mathrm{~mJ} / \mathrm{cm}^{2}$ and $\mathrm{F}_{\mathrm{sat}}=0.293 \mathrm{~mJ} / \mathrm{cm}^{2}$ for $\mathrm{MAPbBr}_{3}$ and $\mathrm{MAPbI}_{3}$ correspondingly. Therefore, the carrier mobilities derived from the $\mathrm{THz}$ emission experiments are $\mu=58 \mathrm{~cm}^{2} / \mathrm{V} \cdot \mathrm{s}$ and $\mu=88 \mathrm{~cm}^{2} / \mathrm{V} \cdot \mathrm{s}$ for $\mathrm{MAPbI}_{3}$ and $\mathrm{MAPbBr}_{3}$. The obtained charge carrier mobilities are in agreement with the data obtained for the studied perovskite materials using conventional methods [28].

We assume that the absorption coefficients for $\mathrm{MAPbI}_{3}$ are 0.94 at $400 \mathrm{~nm}$ and 0.22 at $800 \mathrm{~nm}$ (Figure 3a). Figure 7 shows a comparison of the THz pulse energy and the optical energy and applied bias voltage obtained from the $\mathrm{MAPbI}_{3}$-based LAPCA under above and below the bandgap excitation. In accord with our optical pump/optical probe data in the case of $800 \mathrm{~nm}$, there is a considerably lower carrier generation since the excitation energy is well below the bandgap of both materials. Moreover, under $800 \mathrm{~nm}$ excitation of the $\mathrm{MAPbBr}_{3} \mathrm{LAPCA}$, we were not able to generate any detectable $\mathrm{THz}$ signals.

(a)

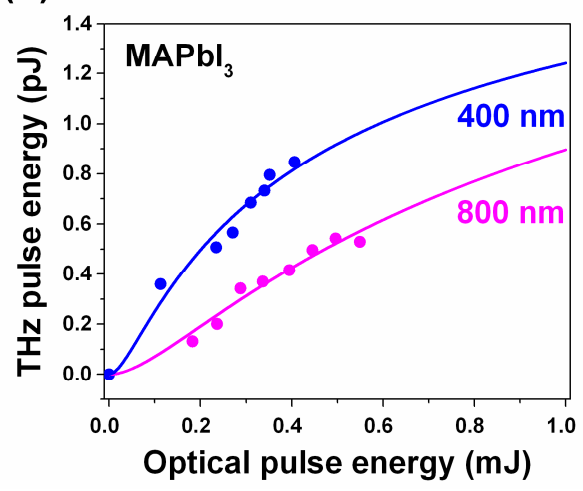

(b)

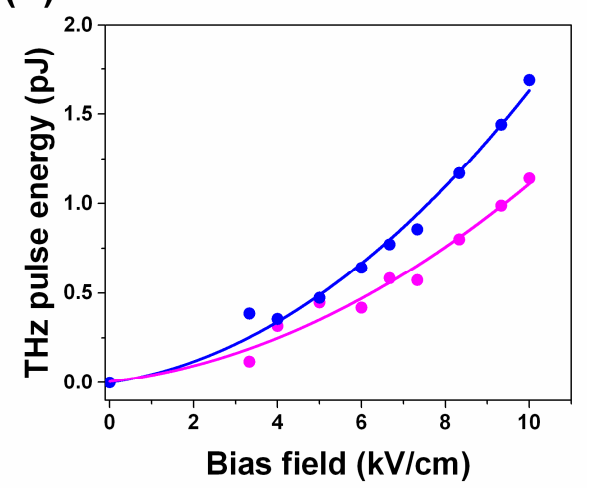

Figure 7. The $\mathrm{MAPbI}_{3}$-based HP-LAPCA terahertz pulse energy as a function of the optical excitation energy (a) and applied bias voltage (b) for pumping above ( $400 \mathrm{~nm}$, blue) and below ( $800 \mathrm{~nm}$, magenta) the bandgap. 


\section{Conclusions}

To summarize, the applicability of solution-processed polycrystalline films of hybrid organic-inorganic perovskites for the development of terahertz radiation emitters is demonstrated. The large-aperture photoconductive antennas fabricated using methylammoniumbased perovskites demonstrate a generation of several picojoule $\mathrm{THz}$ pulses under above and below the bandgap optical excitation. These results make hybrid perovskite materials competitive with conventional semiconductors for THz emission. Moreover, by applying the combination of time-resolved pump-probe spectroscopy and $\mathrm{THz}$ emission experiments, we determine a $\sim 360$ ps carrier lifetime and mobilities of $\mu=58 \mathrm{~cm}^{2} / \mathrm{V} \cdot \mathrm{s}$ and $\mu=88 \mathrm{~cm}^{2} / \mathrm{V} \cdot \mathrm{s}$ in MAPbI3 and MAPbBr3 polycrystalline films, correspondingly. The developed method can be further applied to study the variety of $3 \mathrm{D}$ and $2 \mathrm{D}$ materials having a perovskite structure.

Author Contributions: All authors contributed extensively to the work presented in this paper. Conceptualization, P.A.O.; methodology, P.A.O., P.A.C., V.V.B. (Vladislava V. Bulgakova), and A.A.U.; data collection and analysis, V.V.B. (Vladislava V. Bulgakova), P.A.C., and P.A.O.; V.V.B. (Vladislava V. Bulgakova), P.A.O. and P.A.C.; samples preparation, D.S.G. and S.V.M.; writing-original draft preparation, P.A.O. and V.V.B. (Vladislava V. Bulgakova); supervision, V.V.B. (Vladimir V. Bukin) and P.A.O. All authors have read and agreed to the published version of the manuscript.

Funding: The work was supported by the Ministry of Science and Higher Education of the Russian Federation, Project 075-15-2020-790.

Conflicts of Interest: The authors declare no conflict of interest.

\section{References}

1. Li, J.; Wang, H.; Chin, X.Y.; Dewi, H.A.; Vergeer, K.; Goh, T.W.; Lim, J.W.M.; Lew, J.H.; Loh, K.P.; Soci, C.; et al. Highly Efficient Thermally Co-evaporated Perovskite Solar Cells and Mini-modules. Joule 2020, 4, 1035-1053. [CrossRef]

2. Manspeaker, C.; Venkatesan, S.; Zakhidov, A.; Martirosyan, K.S. Role of interface in stability of perovskite solar cells. Curr. Opin. Chem. Eng. 2017, 15, 1-7. [CrossRef]

3. Luo, L.; Men, L.; Liu, Z.; Mudryk, Y.; Zhao, X.; Yao, Y.; Park, J.M.; Shinar, R.; Shinar, J.; Ho, K.-M.; et al. Ultrafast terahertz snapshots of excitonic Rydberg states and electronic coherence in an organometal halide perovskite. Nat. Commun. 2017, 8, 15565. [CrossRef] [PubMed]

4. Nayak, P.K.; Mahesh, S.; Snaith, H.J.; Cahen, D. Photovoltaic solar cell technologies: Analysing the state of the art. Nat. Rev. Mater. 2019, 4, 269-285. [CrossRef]

5. Makarov, S.; Furasova, A.; Tiguntseva, E.; Hemmetter, A.; Berestennikov, A.; Pushkarev, A.; Zakhidov, A.; Kivshar, Y. Halideperovskite resonant nanophotonics. Adv. Opt. Mater. 2019, 7, 1800784. [CrossRef]

6. Stranks, S.D.; Eperon, G.E.; Grancini, G.; Menelaou, C.; Alcocer, M.J.P.; Leijtens, T.; Herz, L.M.; Petrozza, A.; Snaith, H.J. Electron-Hole Diffusion Lengths Exceeding 1 Micrometer in an Organometal Trihalide Perovskite Absorber. Science 2013, 342, 341-344. [CrossRef]

7. Zhang, B.; Lv, L.; He, T.; Chen, T.; Zang, M.; Zhong, L.; Wang, X.; Shen, J.; Hou, Y. Active terahertz device based on optically controlled organometal halide perovskite. Appl. Phys. Lett. 2015, 107, 093301. [CrossRef]

8. Kumar, A.; Solanki, A.; Manjappa, M.; Ramesh, S.; Srivastava, Y.K.; Agarwal, P.; Sum, T.C.; Singh, R. Excitons in 2D perovskites for ultrafast terahertz photonic devices. Sci. Adv. 2020, 6, eaax8821. [CrossRef]

9. Obraztsov, P.A.; Lyashenko, D.; Chizhov, P.A.; Konishi, K.; Nemoto, N.; Kuwata-Gonokami, M.; Welch, E.; Obraztsov, A.N.; Zakhidov, A. Ultrafast zero-bias photocurrent and terahertz emission in hybrid perovskites. Commun. Phys. 2018, 1, 14. [CrossRef]

10. Guzelturk, B.; Belisle, R.A.; Smith, M.D.; Bruening, K.; Prasanna, R.; Yuan, Y.; Gopalan, V.; Tassone, C.J.; Karunadasa, H.I.; McGehee, M.D.; et al. Terahertz Emission from Hybrid Perovskites Driven by Ultrafast Charge Separation and Strong ElectronPhonon Coupling. Adv. Mater. 2018, 30, 1704737. [CrossRef]

11. Ponseca, C.S.; Arlauskas, A.; Yu, H.; Wang, F.; Nevinskas, I.; Dūda, E.; Vaičaitis, V.; Eriksson, J.; Bergqvist, J.; Liu, X.-K.; et al. Pulsed Terahertz Emission from Solution-Processed Lead Iodide Perovskite Films. ACS Photonics 2019, 6, 1175-1181. [CrossRef]

12. He, Y.; Su, R.; Huang, Y.; Zhou, Y.; Zhao, Q.; Khurgin, J.B.; Xiong, Q.; Xu, X. High-Order Shift Current Induced Terahertz Emission from Inorganic Cesium Bromine Lead Perovskite Engendered by Two-Photon Absorption. Adv. Funct. Mater. 2019, 29, 1904694. [CrossRef]

13. Korolev, V.I.; Pushkarev, A.P.; Obraztsov, P.A.; Tsypkin, A.N.; Zakhidov, A.A.; Makarov, S.V. Enhanced terahertz emission from imprinted halide perovskite nanostructures. Nanophotonics 2019, 9, 187-194. [CrossRef]

14. Liu, X.; Chanana, A.; Huynh, U.; Xue, F.; Haney, P.; Blair, S.; Jiang, X.; Vardeny, Z.V. Circular photogalvanic spectroscopy of Rashba splitting in 2D hybrid organic-inorganic perovskite multiple quantum wells. Nat. Commun. 2020, 11, 323. [CrossRef]

15. Sun, W.; Liu, Y.; Qu, G.; Fan, Y.; Dai, W.; Wang, Y.; Song, Q.; Han, J.; Xiao, S. Lead halide perovskite vortex microlasers. Nat. Commun. 2020, 11, 4862. [CrossRef] [PubMed] 
16. Zhou, Y.; Hua, Y.; Xu, X.; Fan, Z.; Khurgin, J.B.; Xiong, Q. Nonlinear optical properties of halide perovskites and their applications. Appl. Phys. Rev. 2020, 7, 041313. [CrossRef]

17. Lepeshov, S.; Gorodetsky, A.; Krasnok, A.; Rafailov, E.; Belov, P. Enhancement of terahertz photoconductive antenna operation by optical nanoantennas (Laser Photonics Rev. 11(1)/2017). Laser Photonics Rev. 2017, 11, 1770001. [CrossRef]

18. Burford, N.M.; El-Shenawee, M.O. Review of terahertz photoconductive antenna technology. Opt. Eng. 2017, 56, 010901. [CrossRef]

19. Ropagnol, X.; Kovács, Z.; Gilicze, B.; Zhuldybina, M.; Blanchard, F.; Garcia-Rosas, C.M.; Szatmári, S.; Földes, I.B.; Ozaki, T. Intense sub-terahertz radiation from wide-bandgap semiconductor based large-aperture photoconductive antennas pumped by UV lasers. New J. Phys. 2019, 21, 113042. [CrossRef]

20. Hibberd, M.T.; Healy, A.L.; Lake, D.S.; Georgiadis, V.; Smith, E.J.H.; Finlay, O.J.; Pacey, T.H.; Jones, J.K.; Saveliev, Y.; Walsh, D.A.; et al. Acceleration of relativistic beams using laser-generated terahertz pulses. Nat. Photonics 2020, 14, 755-759. [CrossRef]

21. Arkhipov, R.M.; Arkhipov, M.V.; Pakhomov, A.V.; Babushkin, I.; Demircan, A.; Morgner, U.; Rosanov, N.N. Generation of sub cycle terahertz pulses via coherent control of nonlinear medium by femtosecond pulses. J. Phys. Conf. Ser. 2020, 1571, 012009. [CrossRef]

22. You, J.; Hong, Z.; Yang, Y.; Chen, Q.; Cai, M.; Song, T.-B.; Chen, C.-C.; Lu, S.; Liu, Y.; Zhou, H.; et al. Low-temperature solution-processed perovskite solar cells with high efficiency and flexibility. ACS Nano 2014, 8, 1674-1680. [CrossRef] [PubMed]

23. Hebling, J.; Yeh, K.-L.; Hoffmann, M.C.; Bartal, B.; Nelson, K.A. Generation of High-Power Terahertz Pulses by Tilted-Pulse-Front Excitation and Their Application Possibilities. J. Opt. Soc. Am. B 2008, 25, B6-B19. [CrossRef]

24. Obraztsov, P.A.; Rybin, M.G.; Tyurnina, A.V.; Garnov, S.V.; Obraztsova, E.D.; Obraztsov, A.N.; Svirko, Y.P. Broadband LightInduced Absorbance Change in Multilayer Graphene. Nano Lett. 2011, 11, 1540-1545. [CrossRef] [PubMed]

25. Obraztsov, P.A.; Chizhov, P.A.; Kaplas, T.; Bukin, V.V.; Silvennoinen, M.; Hsieh, C.-F.; Konishi, K.; Nemoto, N.; Kuwata-Gonokami, M. Coherent Detection of Terahertz Radiation with Graphene. ACS Photonics 2019, 6, 1780-1788. [CrossRef]

26. Yano, R.; Hattori, T.; Shinojima, H. Improvement of signal-to-noise ratio of terahertz electromagnetic waves by bias field modulation of photoconductive antenna. Jpn. J. Appl. Phys. 2006, 45, 8714-8716. [CrossRef]

27. Yan, H.; An, B.; Fan, Z.; Zhu, X.; Lin, X.; Jin, Z.; Ma, G. Ultrafast terahertz probe of photoexcited free charge carriers in organometal CH3NH3PbI3 perovskite thin film. Appl. Phys. A 2016, 122, 414. [CrossRef]

28. Herz, L.M. Charge-Carrier Mobilities in Metal Halide Perovskites: Fundamental Mechanisms and Limits. ACS Energy Lett. 2017, 2, 1539-1548. [CrossRef]

29. Yamada, Y.; Nakamura, T.; Endo, M.; Wakamiya, A.; Kanemitsu, Y. Photocarrier Recombination Dynamics in Perovskite CH3NH3PbI3 for Solar Cell Applications. J. Am. Chem. Soc. 2014, 136, 11610-11613. [CrossRef]

30. Li, C.; Wang, A.; Deng, X.; Wang, S.; Yuan, Y.; Ding, L.; Hao, F. Insights into Ultrafast Carrier Dynamics in Perovskite Thin Films and Solar Cells. ACS Photonics 2020, 7, 1893-1907. [CrossRef]

31. Bulgakova, V.; Ushakov, A.; Chizhov, P.; Yudin, N.; Zinovev, M.; Podsevalov, S.; Dolmatov, T.; Bukin, V.; Garnov, S. Terahertz generation by means of $\mathrm{ZnGeP2}$ large aperture photo-conductive antenna. Opt. Eng. 2021. accepted.

32. Hafez, H.A.; Chai, X.; Ibrahim, A.; Mondal, S.; Férachou, D.; Ropagnol, X.; Ozaki, T. Intense terahertz radiation and their applications. J. Opt. 2016, 18, 093004. [CrossRef] 